\title{
1 Nestin: a biomarker of aggressive uterine cancers
}

2 Erica R. Hope ${ }^{1,2}$, Paulette Mhawech-Fauceglia ${ }^{3}$, Tanja Pejovic ${ }^{4}$, Christopher M. Zahn ${ }^{5,6}$,

3 Guisong Wang ${ }^{2}$, Thomas P. Conrads ${ }^{2,6,7}$, G. Larry Maxwell ${ }^{2,7,8}$, Chad A. Hamilton ${ }^{1,2,6,7}$,

$4 \quad$ Kathleen M. Darcy ${ }^{2,6-7 ¥}$, Viqar Syed ${ }^{6,7 ¥}$

$6{ }^{1}$ Walter Reed National Military Medical Center, Department of Obstetrics and

7 Gynecology, 8901 Wisconsin Avenue, Bethesda, MD 20889

$8{ }^{2}$ Women's Health Integrated Research Center at Inova Health System, Department of

9 Defense Gynecologic Cancer Center of Excellence, 3289 Woodburn Road, Suites 370

10 and 375, Annandale, VA 22003

$11{ }^{3}$ University of Southern California, Department of Pathology, 1100 N. State Street, Los

12 Angeles, CA 90033

$13{ }^{4}$ Oregon Health \& Science University, Department of Obstetrics and Gynecology, 3181

14 SW Sam Jackson Park Road, L466, Portland, OR, 97239

$15{ }^{5}$ American College of Obstetricians and Gynecologists, 409 125th St SW, Washington,

16 DC 20024

$17{ }^{6}$ Uniformed Services University, Department of Obstetrics \& Gynecology, 4301 Jones

18 Bridge Road, Bethesda, MD 20814

$19{ }^{7}$ John P. Murtha Cancer Center, 8901 Wisconsin Avenue, Bethesda, MD 20889

$20{ }^{8}$ Inova Fairfax Hospital, Department of Obstetrics \& Gynecology, 3300 Gallows Road,

21 Falls Church, VA 22042

22 Key Words: nestin, uterine cancer, TMA, prognostic, predictive, biomarkers,

23 immunohistochemistry 
24 Short Title: Nestin: a biomarker for aggressive uterine cancers

252 Tables, 4 Figures, Supplements S1-S6.

$26 ¥$ Corresponding Authors:

27 Viqar Syed, PhD, Uniformed Services University, Department of Obstetrics \&

28 Gynecology, 4301 Jones Bridge Road, Bethesda, MD 20814, Phone: 301-295-3128, Fax:

29 301-295-6774, Email: viqar.syed@usuhs.edu

30 Kathleen M Darcy, PhD, DoD Gynecologic Cancer Center of Excellence, Women's

31 Health Integrated Research Center at Inova Health System, 3289 Woodburn Road, Suite

32 370, Annandale VA, 22003, Phone: 571-205-2607, FAX: 703-560-0919, Email:

33 DarcyK@WHIRC.org

$34 *$ This abstract was e-published for the American Society of Clinical Oncology 2015

35 Annual Meeting.

37 Disclaimer: The opinions or assertions contained herein are the private views of the

38 authors and are not to be construed as official or as reflecting the views of the

39 Department of the Air Force, the Department of the Army, the Department of the Navy, 40 or the Department of Defense.

41

42 Funding for this project is from The John P. Murtha Cancer Center Award at Walter Reed

43 (MDA905-02-2-0005, PI: Viqar Syed) and the USAMRMC (W81XWH-11-2-013, PI:

44 Chad A. Hamilton). 


\section{ABSTRACT}

46 Objective. Evidence of potential prognostic and predictive value for nestin was

47 investigated in well-annotated uterine cancers (UCs).

48 Methods. Nestin expression and previously-published biomarkers were evaluated by

49 immunohistochemistry (IHC) in UC tissue microarrays. Biomarkers were categorized as

50 low vs. high, and nestin was cut at $10 \%$ positive staining. Relationship between nestin

51 and clinicopathologic factors, biomarkers and outcome were evaluated using exact/log-

52 rank testing or logistic/Cox modeling.

53 Results. There were 323 eligible cases, $34 \%$ had advanced stage disease, 37\% had type II

54 disease, and 5\% were carcinosarcomas. High nestin, observed in 19\% of cases, was more

55 common in advanced vs. early stage disease, type II cancers or uterine carcinosarcoma

56 vs. type I cancers, grade 3 disease, positive lymphovascular space invasion (LVSI) and

57 tumors $>6 \mathrm{~cm}(\mathrm{p}<0.05)$. Nestin was inversely correlated with ER, PR and TFF3, and

58 correlated with p53 and IMP3. Women with high vs. low nestin had worse progression-

59 free survival (PFS) and cancer-specific survival overall, and worse PFS in the subset who

60 received no adjuvant therapy or radiation, or had early stage, type I disease or tumors

61 with both low and high ER, PR, TFF3, PTEN, p53 or IMP3. The relationship between

62 nestin and PFS was independent of stage, LVSI and risk categorization but not type of

63 UC.

64 Conclusions. High nestin was more common in UCs with aggressive features and poor

65 outcome. Nestin may represent a predictive biomarker for treatment selection for patients

66 previously considered to be lower risk and a candidate for no or radiation-based adjuvant

67 therapy, and compliment ER/PR testing. 


\section{INTRODUCTION}

69 Uterine cancer is the fifth leading cause of cancer in women worldwide and the

70 most common gynecologic cancer in the United States, with diagnosis in over 350,000

71 and 54,000, and deaths in 68,000 and 10,000 women each year in the world and U.S.,

72 respectively [1,2]. Since the 1970's, uterine cancer incidence has steadily increased while

73 five-year overall survival has decreased [3]. The obesity epidemic and increasing average

$74 \quad$ lifespans are likely associated with these trends.

75 Uterine cancers are currently classified by histology. The majority of uterine

76 cancers are considered type I disease, have a good prognosis, and are most closely

77 associated with unopposed endogenous or exogenous estrogen [4]. Type II cancers

78 include grade 3 endometrioid, serous, and clear cell [5], and the remaining cancers of the

79 uterine corpus encompass carcinosarcomas and other sarcomas. These non-low-grade

80 endometrioid cancers are typically more virulent and account for a disproportionate

81 number of uterine cancer deaths [6,7]. The Cancer Genome Atlas (TCGA) Research

82 Network and other groups are defining molecular distinctions and similarities between

83 the different uterine cancer subtypes [8] and these biomarkers have been shown to have

84 predictive [9] and biologic value [9-14].

85 Clinical management is based on the above classifications, which take into

86 account histology and grade, in conjunction with known risk factors such as surgical

87 stage, lymphovascular space invasion (LVSI), tumor size, and age at diagnosis [12,15],

88 but no effective therapies exist for aggressive disease. Current risk stratification falls

89 short of preventing over-treatment in many patients who would have never recurred and

90 under-treatment in many who may ultimately die of recurrent or progressive disease. 
91 Given the prevalence of low risk uterine cancer patients, identification of biomarkers that

92 aid in risk stratification are needed.

93 Nestin, a class VI intermediate filament protein first described as a neural stem

94 marker [16], regulates the TGF $\beta$ pathway $[17,18]$ and plays an important role in cancer

95 cell migration, invasion, and metastasis by interacting with vimentin or desmin and

96 modulating the expression of actin and cell adhesion molecules [17,19-21]. In vitro

97 studies have shown that knockdown of nestin inhibits migration, invasion, and metastasis

98 of cancer cells [17,22]. Nestin also plays a key role in angiogenesis [19]. Though

99 unexplored in uterine cancer, nestin expression has been reported in ganglioglioma,

100 ovarian, head and neck, prostate, bladder, and pancreatic cancers, and is a prognostic

101 indicator of poor survival in many cancers [20,21]. In the current investigation, we

102 examined the potential prognostic and predictive value of nestin expression levels in

103 uterine cancer by studying associations with established prognostic clinical factors and

104 biomarkers as well as measures of clinical outcome, including progression-free (PFS) and

105 cancer-specific survival (CSS). 


\section{MATERIALS AND METHODS}

\section{Uterine Cancer Patients with Formalin-Fixed and Paraffin-Embedded Tumor}

108 The University of Southern California archives and database were searched for

109 uterine cancer patients treated from 1998 to 2010. All tissue specimens were collected

110 and medical records reviewed under approved protocols from the Institutional Review

111 Board (IRB). Endometrioid, clear cell, serous, or carcinosarcoma patients with de-

112 identified clinical, outcome, and evaluable nestin data were eligible. Treatment decisions

113 were made by physician and patients. For the purposes of this analysis, stage I \& II

114 disease were categorized as early stage uterine cancer, and stage III \& IV disease were

115 categorized as advanced stage uterine cancer. In addition, grade 1 and 2 (G1/G2)

116 endometrioid carcinomas were categorized as type I uterine cancer. Serous

117 adenocarcinomas, clear cell carcinomas, and grade 3 (G3) endometrioid carcinomas were

118 categorized as type II uterine cancer. Carcinosarcomas including homologous,

119 heterologous or not specified were evaluated collectively as a separate subtype.

120 Risk categorization represented a modification of the Keys GOG-99 and

121 PORTEC classification $[15,23]$ with myometrial invasion categorized at 50\%. The low-

122 risk group included patients with G1 or G2 endometrioid endometrial adenocarcinoma

123 with stage IA disease ( $<50 \%$ myometrial invasion) and negative LVSI. The intermediate-

124 risk patients with stage I or II disease were further subdivided into low- vs. high-

125 intermediate risk groups based on age at diagnosis ( $<50,50$ to $69,70+$ years olds) and

126 other clinical risk factors including positive LVSI, deep myometrial invasion ( $\geq 50 \%)$,

127 and G2/G3 disease. High-intermediate risk patients diagnosed at 70+ years of age needed

128 one other clinical risk factor, at 50 to 69 years of age needed two other clinical risk 
129 factors and at $<50$ years of age needed all three other clinical risk factors. Patients not

130 meeting the high-intermediate risk criteria within the intermediate risk group were

131 classified as low-intermediate risk. The high-risk group included patients with stage

132 III/IV disease as well as those with stage I/II disease with a non-endometrioid histologic

133 subypes such as serous adenocarcinomas (with or without the papillary designation),

134 clear cell carcinomas, and carcinosarcoma. For analysis purposes, patients with low and

135 low-intermediate risk groups were aggregated into a lower risk category, and patients

136 with high-intermediate and high risk were combined into a higher risk category.

\section{Tissue Microarrays}

138 Uterine cancer tissue microarrays (TMAs) were constructed using archival tissue

139 from eligible patients as previously described [24]. Briefly, after carefully selecting the

140 morphologically representative region from the hematoxylin \& eosin (H\&E)-stained

141 section, $0.6 \mathrm{~mm}$ cores were punched from the individual donor formalin-fixed, paraffin-

142 embedded blocks, and incorporated into the TMA paraffin receiver blocks. To account

143 for tumor heterogeneity, core were sampled from three different areas of each tumor. One

144 section from each TMA block was stained with H\&E to confirm the presence of the

145 tumor by light microscopy.

146 Nestin Immunohistochemistry (IHC) Assay and Scoring Criteria

$147 \quad$ Four $\mu \mathrm{m}$ thick sections prepared on positively charged slides from tumor blocks

148 from individual patients or TMA blocks were deparaffinized and pretreated in citrate

149 buffer $\mathrm{pH} 6.0$ for $20 \mathrm{~min}$. Sections were cooled $20 \mathrm{~min}$ and incubated $10 \mathrm{~min}$ at ambient

150 temperature in $3 \% \mathrm{H}_{2} \mathrm{O}_{2}$ to quench endogenous peroxidase activity. Blocking was

151 performed using serum-free protein block, (Dakocytomation, Carpenteria, CA) for 30 
152 min. Slides were loaded on a Dakocytomation autostainer and an anti-nestin antibody

153 (1:50, LifeScience, Memphis, TN; monoclonal) was applied for $1 \mathrm{~h}$. Diaminobenzidine

154 tetrahydrochloride was added for development for $10 \mathrm{~min}$, followed by counterstaining

155 with hematoxylin. Negative control slides omitting the primary antibody were included in 156 all assays.

157 Immunostaining was reviewed using conventional light microscopy and scored by

158 a board-certified gynecologic pathologist (PMF), by intensity and percentage of positive

159 tumor cells. Staining intensity was categorized as $0,1+$ (light brown), 2+ (moderate

160 brown) or $3+$ (dark brown), and by percentage of positive tumor cells categorized as 0 for

161 none, 1 for $\leq 10 \%, 2$ for $11-25 \%, 3$ for $25-50 \%, 4$ for $51-75 \%$ and 5 for $>75 \%$. Cores

162 were not evaluated if the core was lost, severely damaged, and/or did not have sufficient

163 tumor cellularity. The reviewer was blinded to clinical data. Uterine cancer cases were

164 excluded from statistical analysis if less than three cores were available for analysis

165 secondary to inadequate cancer tissue or poor quality of the specimen. Tumor staining for

166 nestin was very consistent across individual full tissue sections and across triplicate cores

167 for individual patients. For purposes of primary analysis, nestin expression was

168 categorized as low ( $\leq 10 \%$ of tumor cells with positive staining) versus (vs.) high (>10\%

169 of tumor cells with positive staining). This cut point was selected based on Western blot

170 expression of nestin in immortalized and malignant endometrial cancer cell lines in vitro

171 showing that low vs. high level expression rather than negative vs. positive expression.

172 Previously Published IHC Biomarkers of Uterine Cancer

173 IHC staining data in these uterine cancer TMAs were available for estrogen

174 receptor (ER), progesterone receptor (PR), trefoil factor 3 (TFF3), phosphatase and tensin 
175 homolog (PTEN), tumor protein p53 (p53), and insulin-like growth factor II mRNA-

176 binding protein (IMP3) $[9,10]$. These biomarkers were initially scored based on overall

177 percent positive tumor cells ( $0=$ none, $1=1-5 \%, 2=6-50 \%, 3=51-100 \%)$ and staining

178 intensity ( 0 for negative; $1+$ for weak; $2+$ for moderate; and $3+$ for strong/dark brown),

179 and then categorized as low vs. high using the following threshold levels -- ER, PR,

180 IMP3 and TFF3 were categorized as low when the additive percent and intensity scores

181 were $\leq 3$ vs. high when the additive score was $>3$. PTEN was categorized as low when

182 percent tumor staining was $\leq 10 \%$ positive tumor cells and intensity was $\leq 1 \mathrm{vs.} \mathrm{high} \mathrm{when}$

$183>10 \%$ of tumor cells were positive and intensity was $2+$. The tumor suppressor p53 was

184 classified as low when percent tumor staining was $\leq 10 \%$ positive tumor cells and

185 intensity was $\leq 2 \mathrm{vs}$. high when $>10 \%$ of tumor cells were positive and intensity was $3+$.

186 Analysis of Public RNASeq Data for Nestin and Other Biomarkers.

187 Normalized level 3 RNASequencing (RNASeq)V2 RSEM expression for nestin,

188 ER, PR, TFF3, p53 and IMP3 with relevant clinical annotations were downloaded from

189 the TCGA Data Portal https://tcga-data.nci.nih.gov/tcga/tcgaDownload.jsp for patients

190 with uterine corpus endometrial carcinomas.

191 Statistical Analyses

192 Statistical analyses were performed using SPSS Version 22 (IBM Corp:

193 Pittsburgh, PA). Relationships between immunohistochemical staining intensity of nestin

194 and clinical characteristics or previously published biomarkers were evaluated with

195 Fisher's exact test and/or logistic regression modeling. Survival analyses for time-to-

196 event data were performed for biomarkers based on IHC staining or RNAseq data using

197 Cox proportional hazard regression modeling with a Wald test, Kaplan-Meier method 
198 with a log-rank test. Assessments of five-year PFS or CSS were evaluated using logistic 199 regression modeling to calculate odds ratios (OR) and 95\% confidence intervals (CI) and 200 generating receiver operator curves (ROC) to calculate area under the curve (AUC) and $20195 \%$ CI. PFS was calculated as the time in months from primary surgery to disease 202 progression or uterine cancer death, or to the date of last contact for women who were 203 alive with no evidence of disease progression or non-cancer death (censored). CSS was 204 calculated as the interval from primary surgery to uterine cancer death or to the date of 205 last contact for women who were alive or died of a cause other than uterine cancer 206 (censored). OS was calculated as the time from primary surgery to death or to the date of 207 last contact for those who were still alive (censored). Censoring of events was performed 208 at 5 years after primary surgery. All reported p-values are two-sided with statistical 209 significance set at $\mathrm{p}<0.05$. Significance levels with $\mathrm{p}<0.007$ passed a conservative 210 Bonferroni correction for multiple testing for 7 biomarkers. Hazard ratio (HR) for Cox 211 regression modeling and OR for logistic regression modeling are presented when 95\% 212 confidence intervals (CI) did not overlap 1.0. 


\section{RESULTS}

214 The characteristics for the 323 eligible uterine cancer patients are presented in

215 Table 1 and with additional details in Supplement S1. Median age of the patients at the

216 time of primary cancer surgery was 63 years. The distribution of cases by stage was

$21755.7 \%$ with stage I disease, $10.2 \%$ stage II, $22.6 \%$ stage III, and $11.5 \%$ stage IV. Fifty-

218 seven percent of cases had type I disease (G1 or G2 endometrioid endometrial cancer),

$21937.5 \%$ had type II disease (serous or clear cell carcinomas or grade 3 endometrioid

220 endometrial carcinoma), and 5.3\% were carcinosarcomas of the uterine corpus. Thirty

221 percent had tumors $6+\mathrm{cm}$ in size, and 33\% exhibited LSVI. Median follow-up was 44

222 months with 64 (20.1\%) cases with disease progression, 56 (17.3\%) deaths attributable to

223 uterine cancer, $22(6.8 \%)$ non-cancer deaths, and $71.8 \%$ of patients received adjuvant

224 therapy.

225 Tumor Nestin Expression in Uterine Cancer Patients

226 Uterine cancer patients were classified based on low [Figure (Fig.) 1A] or high

227 (Fig. 1B) tumor expression of nestin. High IHC staining for nestin was observed in 19\%

228 of cases overall. High nestin expression was associated with advanced stage, type II

229 cancers or carcinosarcomas, non-endometrioid cell type, high grade, large tumor size,

230 presence of LVSI, but not age of diagnosis as either a continuous or categorical variable

231 (Table 1). High expression of nestin was also more common in each of these subgroups

232 (Supplement S2). The relationships between nestin and tumor stage, type of uterine

233 cancer and risk categorization were also examined in greater detail. High nestin was

234 observed in $10 \%$ of stage IA, $23 \%$ of stage IB, $24 \%$ of stage II, $30 \%$ of stage III and $27 \%$

235 of stage IV tumors ( $\mathrm{p}=0.002$ ) or in $6 \%$ of $\mathrm{G} 1$ endometrioid, $13 \%$ of $\mathrm{G} 2$ endometrioid, 
$23622 \%$ of G3 endometrioid, $25 \%$ of clear cell and $40 \%$ of serous carcinomas as well as $61 \%$

237 of carcinosarcomas $(\mathrm{p}<0.001)$, respectively. Higher risk group patients were 5.6 times

238 more likely to exhibit high tumor expression of nestin than the low and low-intermediate

239 risk subgroups $(\mathrm{p}<0.001)$.

$240 \quad$ IHC Staining of Nestin and Other Biomarkers of Uterine Cancer

241 Type I endometrial tumors typically exhibit high ER, PR, TFF3, and loss of

242 PTEN. Nestin expression was inversely associated with ER, PR, and TFF3, but not PTEN

243 (Table 2 and Supplement S3). Type II endometrial tumors are often characterized by

244 high p53 or IMP3 expression. High nestin expression was more common in type II

245 disease and tumors with high p53 or high IMP3 (Table 2 and Supplement S3).

246 IHC Staining for Nestin a Biomarker of Poor Clinical Outcome

247 The relationship between nestin and clinical outcome was evaluated for potential

248 prognostic, independent prognostic, or predictive value. High nestin expression was

249 associated with worse PFS (Fig. 2A), CSS (Supplement S4), and overall survival (data

250 not shown). Women with high vs. low tumor expression of nestin had an increased risk of

251 disease progression $(\mathrm{HR}=2.787,95 \% \mathrm{CI}=1.798-4.320, \mathrm{p}<0.001)$, cancer death

$252(\mathrm{HR}=2.325,95 \% \mathrm{CI}=0.323-4.086, \mathrm{p}=0.003)$, and death due to any cause $(\mathrm{HR}=1.791$,

$25395 \% \mathrm{CI}=1.057-3.035, \mathrm{p}=0.03)$.

254 High tumor expression of nestin was not an independent predictor of PFS or CSS

255 after adjusting for age, stage, and an aggregate of cell type and grade in the same model.

256 Individual adjusted models (Supplement S5) demonstrated that the relationship between

257 nestin and PFS was maintained after adjustment by treatment (adjusted HR=1.975), stage

258 (adjusted HR=2.006), LVSI (adjusted HR=1.984) or a modified risk categorization based 
259 on GOG-99 and PORTEC criteria (adjusted HR=1.878) each with 95\% CIs that did not

260 overlap 1, but not by the cell type and/or grade (95\% CIs overlap 1.0).

261 Logistic regression modeling and receiver operator curve (ROC) analyses

262 provided measures of predictive accuracy and demonstrated that high versus (vs) low

263 nestin was associated with an increased risk of disease progression within 5-years

$264(\mathrm{OR}=3.450,95 \% \mathrm{CI}=1.942-6.129)$. The presence of high nestin predicted risk of 5-year

265 PFS with an AUC of 0.608 (95\% CI=0.535-0.681). Incorporation of nestin with stage and

266 uterine cancer type improved the prediction of 5-year PFS to an AUC of 0.801 (95\%

$267 \mathrm{CI}=0.752-0.850$ ). The accuracy for predicting 5-year PFS dropped to 0.624 with ER

268 (95\% CI=0.544-704), 0.566 with ER and PR (95\% CI=0.482-0.650) or 0.568 with ER,

269 PR and p53 (95\% CI=0.484-0.652) added to the model with nestin, stage, uterine cancer

270 type.

271 IHC Staining of Other Biomarkers and PFS

272 The relationship between the previously published biomarkers and PFS censored

273 at 60 months was also assessed. Better PFS was observed in women with high vs. low

274 ER, PR or TFF3 (Fig. 2 B-D, log-rank p<0.001), but not PTEN expression. Worse PFS

275 was seen in women with high vs. low p53 or IMP3 (Fig. 2E-F, log-rank p $\leq 0.01$ ).

276 Relationship between IHC Staining of Nestin and Treatment on PFS

277 Subset analyses were performed to examine the relationship between nestin and

278 PFS in greater detail. The first of these analyses examined the impact of nestin expression

279 and treatment on PFS. Poor PFS was observed in women with high vs low nestin who

280 received no or radiation-based adjuvant therapy (Fig. 3A-B, log rank p<0.001). Nestin 
281 expression did not stratify PFS in the subset of patients who received chemotherapy or

282 chemoradiation (Fig. 3C).

283 Relationship between Nestin and Prognostic Clinical Variables on PFS

284 The combination of nestin and prognostic clinical variables such as stage, LVSI,

285 risk categorization (defined in methods), and type of uterine cancer (cell type by grade)

286 on PFS was also assessed. Women with high nestin expression had worse PFS than those

287 with low nestin expression in the subset with G1 or G2 endometrioid carcinoma, early

288 stage disease, negative LVSI, lower risk, and higher risk patients (Fig. 3D-H). The level

289 of nestin expression did not stratify PFS in the subset of women with G3 endometrioid

290 carcinomas, non-endometrioid histologies, advanced stage or positive LVSI

291 (Supplement S6A-D).

292 Relationship between Nestin and Other Biomarkers on PFS

293 The combined effect of nestin with type I biomarkers or type II biomarkers on

294 PFS was also investigated. Categorized tumor expression of nestin consistently stratified

295 PFS overall and when crossed with the other biomarkers. Women with high relative to

296 low tumor expression of nestin consistently had worse PFS in the subset with low and

297 with high ER (Fig. 4A-B), PR (Fig. 4C-D), p53 (Fig. 4E-F), PTEN (Supplement S7A-

298 B), TFF3 (Supplement S7C-D) or IMP3 (Supplement S7E-F), respectively.

299 Transcript Expression of Nestin or Other Biomarkers and PFS

300 Data from the TCGA uterine cancer project was utilized to determine if transcript

301 expression of nestin (or other biomarkers) had a similar relationship with PFS as

302 observed using IHC-derived data or not (Supplements S8-S10). High vs. low tumor

303 staining of either nestin or IMP3 was associated with worse PFS and an increased risk of 
304 disease progression but neither of these biomarkers exhibited prognostic value at the

305 RNA-seq level. High vs. low tumor staining of p53 was associated with worse PFS

306 especially in type II cancers whereas higher transcript expression of TP53 was associated

307 with better PFS and a reduced risk of disease progression in uterine cancers and the

308 subset of endometrioid but not serous cancers. Consistent with IHC-based results, ERS1,

$309 P G R$ and TFF3 were associated with better PFS and a reduced risk of disease progression

310 in uterine cancers. 


\section{DISCUSSION}

312 In our study, high nestin expression was more common in advanced stage, non-

313 endometrioid subtypes, positive LVSI, tumor size $6+\mathrm{cm}$, and women with disease

314 progression or cancer death. Women with high vs. low nestin had worse progression-free

315 survival (PFS) and cancer-specific survival (CSS) overall, and worse PFS in the subset

316 who received no or radiation-based adjuvant therapy or had early stage, type I disease,

317 and biologically appropriate relationship with conventional biomarkers. Nestin was an

318 independent prognostic factor for PFS or CSS after controlling for stage, LVSI, or risk

319 categorization, but not after correction for type of uterine cancer (cell type and grade).

320 The accuracy of predicting disease progression at five years was $60 \%$ for nestin alone and

$32180 \%$ when nestin was combined with stage and uterine cancer type.

322 Nestin is a regulator of TGF- $\beta[17,18]$ and in vitro experiments have

323 demonstrated that it plays a role in invasion, metastasis, and angiogenesis [17,22]. The

324 association of nestin expression with prognosis has been demonstrated in ganglioglioma,

325 ovarian, head and neck, prostate, bladder, and pancreatic cancer [20,22,25,26], and from

326 the present work, in uterine cancer. Nestin may have utility as a theragnostic triage

327 companion test and opportunity for further risk stratification for clinical management.

328 This may play a particularly important role in patients who may not receive adjuvant

329 therapy but may actually be at a higher risk of recurrence than previously thought due to

330 high nestin expression. The decrease in PFS was also seen in patients treated with

331 adjuvant radiation, but not among patients treated with chemotherapy alone or

332 chemoradiation as these patients are among the highest risk for recurrence. High nestin

333 was more common in tumors with aggressive histologic features, but portended worse 
334 PFS in the lower risk cancers. We speculate that nestin was not able to stratify PFS in

335 women had advanced stage disease or G3 endometrioid endometrial cancer or non-

336 endometrioid cancers as we lack effective therapies for these subsets. This hypothesis is

337 supported by the observation that similar PFS was observed in women who received

338 adjuvant chemotherapy or chemoradiation.

339 One hallmark of invasive and metastatic cancer is the induction of epithelial-

340 mesenchymal transition (EMT) [27-29]. Tumor EMT is a phenotypic switch that

341 promotes the acquisition of a fibroblastoid-like morphology by epithelial tumor cells,

342 resulting in enhanced tumor cell motility and invasiveness, increased metastatic

343 propensity, and resistance to chemotherapy [27,29]. Tumor cells undergoing EMT secrete

344 specific factors, including cytokines, chemokines, and growth factors, such as interleukin

345 (IL)-1, tumor necrosis factor alpha (TNF- $\alpha$ ), and TGF- $\beta$, that promote tumor progression $346[28,29]$.

347 Progesterone has a fundamental role in reproductive biology, uterine cancer

348 treatment, and has been implicated as a chemopreventive agent, for a number of cancers

349 including endometrial cancer [30-32]. A potential key mechanism of the therapeutic and

350 chemopreventive activity involves the progesterone-dependent downregulation of the

351 transforming growth factor-beta (TGF- $\beta$ ) pathway, a driver of cancer cell viability and

352 invasion. Progestin is a therapeutic option for endometrial cancer patients who are poor

353 surgical candidates or wish to maintain fertility [12,33], has a protective effect on the

354 endometrium, and is also being explored for chemopreventive activity alone or in

355 combination with vitamin D [17]. Progestin induces cellular differentiation, the

356 mechanism by which includes suppression of TGF- $\beta$ signaling and inhibition of EMT 
$357[9,10]$. Studies are underway to determine the relationship between progestin signaling

358 and nestin. Future uses of nestin may include evaluation as a surrogate biomarker and in

359 the development of chemopreventive combinations, such as with progestin, especially in

360 patients with multiple risk factors for uterine cancer development (e.g. Lynch syndrome,

361 obesity, chronic anovulation).

362 High nestin expression did not retain independent prognostic relevance after

363 correction for cell type and grade, but did stand up to adjustments for clinical features not

364 always readily apparent at the time of diagnosis. The observation that nestin expression

365 stratified both PFS and CSS overall and in all subsets of lower risk patients illustrates the

366 potential utility of nestin in triaging the apparent low risk patients for more aggressive

367 treatment. The predictive value of nestin was optimal when combined with stage and

368 uterine cancer cell type with accuracy levels achieving $80 \%$. Predictive assessments in

369 the subsets of patients receiving no or radiation therapy has the potential to benefit a

370 significant proportion of uterine cancer patients in the US and globally each year.

371 Biomarkers assist in clinical management decision making of uterine cancer

372 patients, distinguishing those cancers with more or less aggressive features [9-14,33].

373 Markers of type I disease (ER, PR, and TFF3) were inversely associated with nestin

374 expression and, conversely, type II biomarkers (p53 and IMP3) were more common with

375 high nestin expression. Nestin stratified PFS in tumors either with low or with high ER,

376 PR, TFF3, PTEN, p53 and IMP3. This profile of PFS stratification, particularly in

377 patients with type I disease that receive no or adjuvant radiation therapy, offers an

378 opportunity for nestin to act as a clinical biomarker along with ER and PR to more

379 effectively stratify risk in these individuals. Given that type I disease accounts for the 
380 majority of uterine cancers [1-3], the potential impact on women in the US and the world

381 is high. Independent validation will be required to document the true clinical value of

382 nestin in triaging the lowest risk uterine cancer patients with the worst PFS.

383 Development and deployment of robust companion biomarker assays with prognostic and

384 predictive value is needed to enhance the personalized clinical management of uterine

385 cancer patients beyond that provided by estrogen receptor or progesterone receptor 386 (ER/PR).

387 Some biomarkers are coordinately regulated at the transcript and protein levels

388 whereas others are not. There are numerous post-transcriptional, translational and post-

389 translational mechanisms [34,35] that may explain, at least in part, any disparity in the

390 relationship between IHC staining and PFS vs. transcript expression and PFS. In

391 addition, the confounding effect of tumor purity on transcript expression and

392 disparities with IHC staining demonstrated in an assessment of $>10,000$ samples

393 across 21 cancer types from TCGA [36] provide a potential alternate or

394 complimentary explanation for the observed inconsistencies between IHC- vs.

395 RNASeq-based results.

396 High nestin expression is more common in aggressive uterine cancers, but appears

397 to be more predictive of poor outcome in patients with lower risk, including those with

398 early stage disease and G1/G2 endometrioid histologies with and without LVSI. These

399 findings are consistent with the mechanistic studies of nestin and clinical outcome

400 assessments of nestin expression in other cancers $[20,22,25,26]$. It is a testable and

401 biologically relevant protein that may have utility as a triage tool to identify lower risk

402 patients who may require more aggressive treatment. Additional studies will be required 
Hope et al. 20

403 to more thoroughly assess the prognostic, predictive, and theragnostic potential of nestin 404 and to optimize its deployment in therapeutic and chemopreventive settings.

405

406 


\section{REFERENCES}

410 [1] Siegel RL, Miller KD, Jemal A. Cancer statistics, 2015. CA Cancer Journal for

$411 \quad$ Clinicians 2015;65:5-29.

412 [2] Fitzmaurice C, Dicker D, Pain A, Hamavid H, Moradi-Lakeh M, MacIntyre MF,

$413 \quad$ Naghavi M et al. The Global Burden of Cancer 2013. JAMA Oncol 2015:505-

$414 \quad 27$.

415 [3] Carlson MJ, Thiel KW, Yang S, Leslie KK. Catch it before it kills: Progesterone, 416 obesity, and the prevention of endometrial cancer. Discov Med 2012;14:215-

$417 \quad 22$

418 [4] Reid-Nicholson M, Iyengar P, Hummer AJ, Linkov I, Asher M, Soslow RA.

419 Immunophenotypic diversity of endometrial adenocarcinomas: implications

420 for differential diagnosis. Mod Pathol 2006:1091-110.

421 [5] Alkushi A, Köbel M, Kalloger SE, Gilks CB. High-grade endometrial

422 carcinoma: Serous and grade 3 endometrioid carcinomas have different

423 immunophenotypes and outcomes. International Journal of Gynecological

$424 \quad$ Pathology 2010;29:343-50.

425 [6] Endometrial cancer. ACOG Pract Bull No. 149. American College of

$426 \quad$ Obstetricians and Gynecologists; 2015. Available from

427 http://www.acog.org/from_home/publications/misc/pb149.htm. Accessed

$428 \quad$ on July $22,2015$.

429 [7] Hamilton CA, Cheung MK, Osann K, Chen L, Teng NN, Longacre TA, Powell

430 MA, Hendrickson MR, Kapp DS, Chan JK. Uterine papillary serous and clear

$431 \quad$ cell carcinomas predict for poorer survival compared to grade 3 
endometrioid corpus cancers. Br J Cancer 2006;94:642-46.

433 [8] Getz G, Gabriel SB, Cibulskis K, Lander E, Sivachenko A, Sougnez C, et al.

434 Integrated genomic characterization of endometrial carcinoma. Nature $435 \quad 2013 ; 497: 67-73$.

436 [9] Mhawech-Fauceglia P, Yan L, Liu S, Pejovic T. ER +/PR +/TFF3 +/IMP3 -immunoprofile distinguishes endometrioid from serous and clear cell carcinomas of the endometrium: a study of 401 cases. Histopathology 2013;62:976-85.

440 [10] Mhawech-Fauceglia P, Wang D, Kim G, Sharifian M, Chen X, Liu Q, Lin YG, Liu

S, Pejovic T. Expression of DNA repair proteins in endometrial cancer predicts disease outcome. Gynecologic Oncology 2014;132:593-8.

443 [11] Mhawech-Fauceglia P, Wang D, Samrao D, Liu S, duPont NC, Pejovic T.

Trefoil factor family 3 (TFF3) expression and its interaction with estrogen receptor (ER) in endometrial adenocarcinoma. Gynecologic Oncology 2013;130:174-80.

[12] Creasman W, DiSaia M. Chapter 5 - Adenocarcinoma of the Uterine Corpus. $448 \quad$ Eighth Edition. Elsevier Inc; 2012.

449 [13] Lawrenson K, Pakzamir E, Liu B, Lee JM, Delgado MK, Duncan K, Gayther SA, Liu S, Roman L, Mhawech-Fauceglia P. Molecular analysis of mixed endometrioid and serous adenocarcinoma of the endometrium. PLoS ONE 2015;10:e0130909-12.

453 [14] Singh M, Darcy KM, Brady WE, Clubwala R, Weber Z, Rittenbach JV, Akalin A, 454 Whitney CW, Zaino R, Ramirez NC, Leslie KK. Cadherins, catenins and cell 
cycle regulators: Impact on survival in a Gynecologic Oncology Group phase

II endometrial cancer trial. Gynecologic Oncology 2011;123:320-28.

457 [15] Keys HM, Roberts JA, Brunetto VL, Zaino RJ, Spirtos NM, Bloss JD, Pearlman

A, Maiman MA, Bell JG. A phase III trial of surgery with or without adjunctive

external pelvic radiation therapy in intermediate risk endometrial

adenocarcinoma: a Gynecologic Oncology Group study. Gynecologic Oncology 2004;92:744-51.

462 [16] Duggal N, Hammond RR. Nestin expression in ganglioglioma. Exp Neurol 2002;174:89-95.

464 [17] Matsuda Y, Naito Z, Kawahara K, Nakazawa N, Korc M, Ishiwata T. Nestin is a 465 novel target for suppressing pancreatic cancer cell migration, invasion and metastasis. Cancer Biology \& Therapy 2014;11:512-23.

467 [18] Su HT, Weng CC, Hsiao PJ, Chen LH, Kuo TL, Chen YW, Kuo KK, Cheng KH. Stem cell marker nestin is critical for TGF- 1-mediated tumor progression in pancreatic cancer. Molecular Cancer Research 2013;11:768-79.

470 [19] Liang Z-W, Wang Z, Chen H, Li C, Zhou T, Yang Z, Yang X, Yang Y, Gao G, Cai W. Nestin-mediated cytoskeletal remodeling in endothelial cells: novel mechanistic insight into VEGF-induced cell migration in angiogenesis. Am J Physiol Cell Physiol 2015;308:C349-58.

474 [20] Ryuge S. Prognostic Significance of Nestin Expression in Resected Non-small 475 Cell Lung Cancer. Chest 2011;139:862-68.

476 [21] Tabata K-I, Matsumoto K, Minami S, Ishii D, Nishi M, Fujita T, Saegusa M, 
survival after radical cystectomy in patients with urothelial carcinoma of the bladder. PLoS ONE 2014;9:e91548.

480 [22] Kleeberger W, Bova GS, Nielsen ME, Herawi M, Chuang AY, Epstein JI, 481 Berman DM. Roles for the stem cell associated intermediate filament nestin in prostate cancer migration and metastasis. Cancer Research 2007;67:9199-206.

484 [23] Nout RA, Smit V, Putter H, Jürgenliemk-Schulz IM, Jobsen JJ, Lutgens L, van 485 der Steen-Banasik EM, Mens JWM, Slot A, Stenfert Kroese MC, van Bunningen BNFM, Ansink AC, van Putten WLJ, Creutzberg CL. Vaginal brachytherapy versus pelvic external beam radiotherapy for patients with endometrial cancer of high-intermediate risk (PORTEC-2): an open-label, non-inferiority, randomised trial. The Lancet 2010;375:816-23

490 [24] Kononen J, Bubendorf L, Kallioniemi A, Barlund M, Schraml P, Leighton S, 491 Torhorst J, Mihatsch MJ, Sauter G, Kallioniemi OP. Tissue microarrays for high-throughput molecular profiling of tumor specimens. Nature Medicine 1998;4:844-47.

494 [25] Shan Y-S, Chen Y-L, Lai M-D, Hsu H-P. Nestin predicts a favorable prognosis in early ampullary adenocarcinoma and functions as a promoter of metastasis in advanced cancer. Oncol Rep 2014;33:40-8.

497 [26] Sabet MN, Rakhshan A, Erfani E, Madjd Z. Co-Expression of Putative Cancer 498 Stem Cell Markers, CD133 and Nestin, in Skin Tumors. Asian Pacific Journal 499 of Cancer Prevention 2014;15:8161-69.

500 [27] Thiery JP, Sleeman JP. Complex networks orchestrate epithelial- 
mesenchymal transitions. Nat Rev Mol Cell Biol 2006;7:131-42.

502 [28] Yang J, Weinberg RA. Epithelial-Mesenchymal Transition: At the crossroads

503 of development and tumor metastasis. Developmental Cell 2008;14:818-29.

504 [29] Mirantes C, Espinosa I, Ferrer I, Dolcet X, Prat J, Matias-Guiu X. Epithelial-to-

505 mesenchymal transition and stem cells in endometrial cancer. Human

$506 \quad$ Pathology 2013;44:1973-81.

507 [30] Bokhari AA, Lee LR, Raboteau D, Hamilton CA, Maxwell GL, Rodriguez GC, 508 Syed V. Progesterone inhibits endometrial cancer invasiveness by inhibiting the TGFbeta pathway. Cancer Prev Res 2014;7:1045-55.

510 [31] Lee LR, Teng P-N, Nguyen H, Hood BL, Kavandi L, Wang G, Turbov JM, Thaete LG, Hamilton CA, Maxwell GL, Rodriguez GC, Conrads TC, Syed V. Progesterone enhances calcitriol antitumor activity by upregulating vitamin

513 D receptor expression and promoting apoptosis in endometrial cancer cells.

$514 \quad$ Cancer Prev Res 2013;6:731-43.

515 [32] Nguyen H, Ivanova VS, Kavandi L, Rodriguez GC, Maxwell GL, Syed V. Progesterone and 1,25-dihydroxyvitamin D(3) inhibit endometrial cancer cell growth by upregulating semaphorin 3B and semaphorin 3F. Molecular Cancer Research 2011;9:1479-92.

519 [33] Zaino RJ, Brady WE, Todd W, Leslie K, Fischer EG, Horowitz NS, Mannel RS, 520 Walker JL, Ivanovic M, Duska LR. Histologic effects of medroxyprogesterone

521 acetate on endometrioid endometrial adenocarcinoma: a Gynecologic Oncology Group study. International Journal of Gynecological Pathology 2014;33:543-53. 
524 [34] Van Roey K, Davey NE. Motif co-regulation and co-operativity are common 525 mechanisms in transcriptional, post-transcriptional and post-translational 526 regulation. Cell Commun Signal 2015;13:1-16.

527 [35] Cell and Molecular Biology: Concepts and Experiments by Gerald Karp and 528 James G. Patton. 7th Edition John Wiley \& Sons, Inc. 2013,, 514-544.

529 [36] Aran D, Sirota M, Butte AJ. Systemtic pan-cancer analysis of tumor purity. $530 \quad$ Nature Communications. 2015;6:8791. 
Table 1. Clinical characteristics in eligible uterine cancer patients and relationship with tumor expression of nestin.

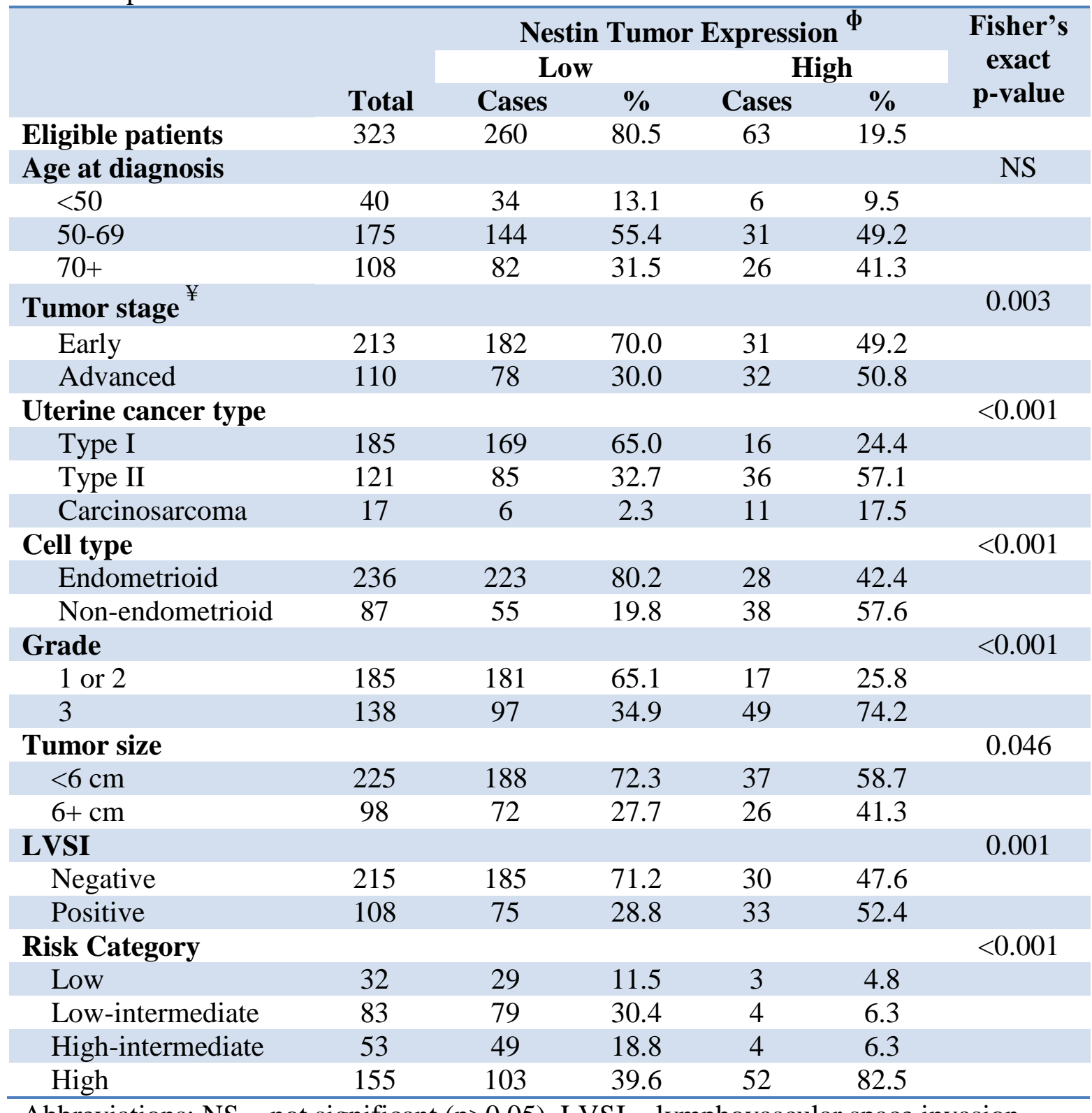

540 Abbreviations: NS $=$ not significant $(\mathrm{p}>0.05), \mathrm{LVSI}=$ lymphovascular space invasion

$541 \phi$ Nestin expression was categorized as low ( $\leq 10 \%$ positive tumor cells) or high $(>10 \%$

542 positive tumor cells).

$543 ¥$ Early represents stage I and II disease. Advanced includes stage III and IV disease. See

544 methods for the definitions for the risk categorization. 
Table 2. Relationship between nestin and other biomarkers in the uterine cancers.

\begin{tabular}{|c|c|c|c|c|c|}
\hline & \multicolumn{4}{|c|}{ Nestin Tumor Expression ${ }^{\phi}$} & \multirow{3}{*}{$\begin{array}{l}\text { Fisher's } \\
\text { exact } \\
\text { p-value }\end{array}$} \\
\hline & \multicolumn{2}{|c|}{ Low } & \multicolumn{2}{|c|}{ High } & \\
\hline & Cases & $\%$ & Cases & $\%$ & \\
\hline \multicolumn{6}{|c|}{ Type I Biomarkers } \\
\hline ER & & & & & 0.001 \\
\hline Low & 81 & 33.9 & 35 & 59.3 & \\
\hline High & 158 & 66.1 & 24 & 40.7 & \\
\hline PR & & & & & $<0.001$ \\
\hline Low & 81 & 33.5 & 39 & 67.2 & \\
\hline High & 161 & 66.5 & 19 & 32.8 & \\
\hline TFF3 & & & & & $<0.001$ \\
\hline Low & 78 & 35.1 & 37 & 63.8 & \\
\hline High & 144 & 64.9 & 21 & 36.2 & \\
\hline PTEN & & & & & NS \\
\hline Low & 152 & 63.3 & 33 & 55.0 & \\
\hline High & 88 & 36.7 & 27 & 45.0 & \\
\hline \multicolumn{6}{|c|}{ Type II Biomarkers $^{¥}$} \\
\hline p53 & & & & & 0.009 \\
\hline Low & 144 & 59.3 & 24 & 40.0 & \\
\hline High & 99 & 40.7 & 36 & 60.0 & \\
\hline IMP3 & & & & & 0.003 \\
\hline Low & 181 & 76.1 & 32 & 55.2 & \\
\hline High & 57 & 23.9 & 26 & 44.8 & \\
\hline
\end{tabular}

546 Abbreviations: NS = not significant ( $\mathrm{p}>0.05$ ); ER (estrogen receptor); PR (progesterone

547 receptor); TFF3 (trefoil factor 3); PTEN (phosphatase and tensin homolog); p53 (tumor

548 protein p53); IMP3 (insulin-like growth factor II mRNA-binding protein 3).

$549 \Phi$ Nestin expression was categorized as low ( $\leq 10 \%$ positive tumor cells) or high ( $>10 \%$

550 positive tumor cells).

$551 ¥$ See methods for details regarding the scoring and categorization of each biomarker. 
554 Figure 1. Tumor expression of nestin and relationship with clinical outcome.

555 Immunohistochemical expression of nestin was categorized as low ( $\leq 10 \%$ positive tumor

556 cells) or high (>10\% positive tumor cells) and representative photomicrographs provided

557 for low (A) and high (B) staining in endometrioid endometrial carcinomas. Lower power

558 images provided in the upper right corner of panels A-B.

559 Figure 2. The relationship between other biomarkers and progression-free survival.

560 Progression-free survival (A) plot censored at 60 months were generated using Kaplan-

561 Meier method, and survival distributions were compared using log-rank test for women

562 with low (blue line) vs. high (green line) tumor expression of nestin. Hazard ratio (HR)

563 and 95\% confidence interval (CI) were generated from univariate Cox regression

564 modeling for progression-free survival (A). Progression-free survival plots censored at

56560 months were generated using Kaplan-Meier method and survival distributions were

566 compared using log-rank test for women with low (blue line) vs. high (green line) tumor

567 expression of ER (B, p<0.001), PR (C, p<0.001), TFF3 (D, <0.001), p53 (E, p=0.01),

568 IMP3 (F, p <0.001). Abbreviations: ER (estrogen receptor); PR (progesterone receptor);

569 TFF3 (trefoil factor 3); p53 (tumor protein p53); IMP3 (insulin-like growth factor II

570 mRNA-binding protein 3). See methods for details regarding the scoring and

571 categorization of each biomarker.

572 Figure 3. Combination of nestin and prognostic clinical variables on progression-

573 free survival. Progression-free survival plots censored at 60 months were generated

574 using Kaplan-Meier method and survival distributions were compared using log-rank test

575 for the subset of women with low (blue line) vs. high (green line) tumor expression of 
576 nestin who received no adjuvant treatment $(\mathbf{A}, \mathrm{p}<0.001)$, radiation $(\mathbf{B}, \mathrm{p}<0.001)$ or

577 chemotherapy/chemoradiation $(\mathbf{C}, \mathrm{p}>0.05)$, or had $\mathrm{G} 1$ or $\mathrm{G} 2$ endometrioid endometrial

578 cancer $(\mathbf{D}, \mathrm{p}<0.001)$, early stage disease $(\mathbf{E}, \mathrm{p}<0.001)$, negative LVSI $(\mathbf{F}, \mathrm{p}=0.001)$, lower

579 risk disease $(\mathbf{G}, \mathrm{p}<0.001)$ or higher risk disease $(\mathbf{H}, \mathrm{p}=0.03)$. Early stage includes stage I

580 and II disease. LVSI refers to lymphovascular space invasion. See methods for

581 definitions for the risk categorizations.

582 Figure 4. Combination of nestin and other biomarkers on progression-free survival.

583 Progression-free survival plots censored at 60 months were generated using Kaplan-

584 Meier (KM) method and survival distributions were compared using pairwise log-rank

585 test for the subset of women categorized by low (blue line) or high (green line)

586 expression of nestin and by either low ER (A), high ER (B), low PR (C), high PR (D),

587 low p53 (E) or high p53 (F). Abbreviations: ER (estrogen receptor); PR (progesterone

588 receptor); p53 (tumor protein p53). See methods for details regarding the scoring and

589 categorization of each biomarker. 
Hope et al. 33

\section{$591 \quad$ Figure 1.}
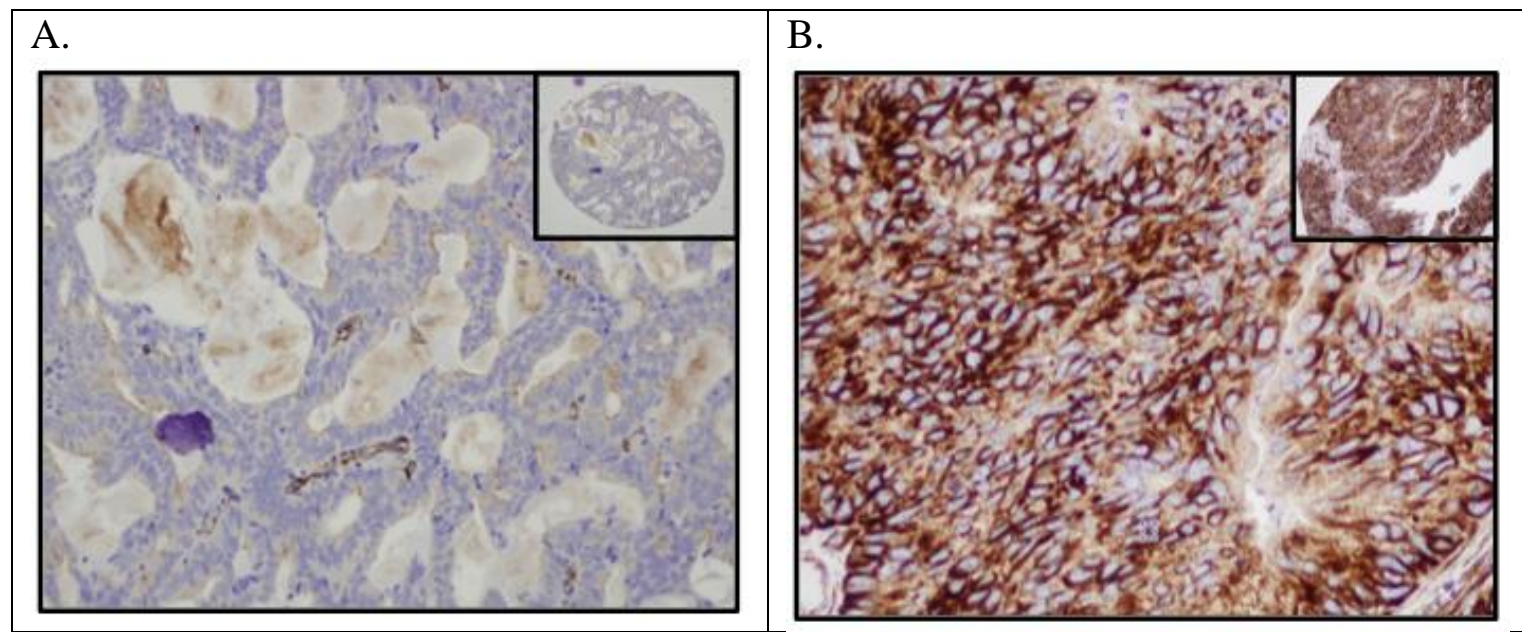

592 
Hope et al. 34

593

Figure 2.

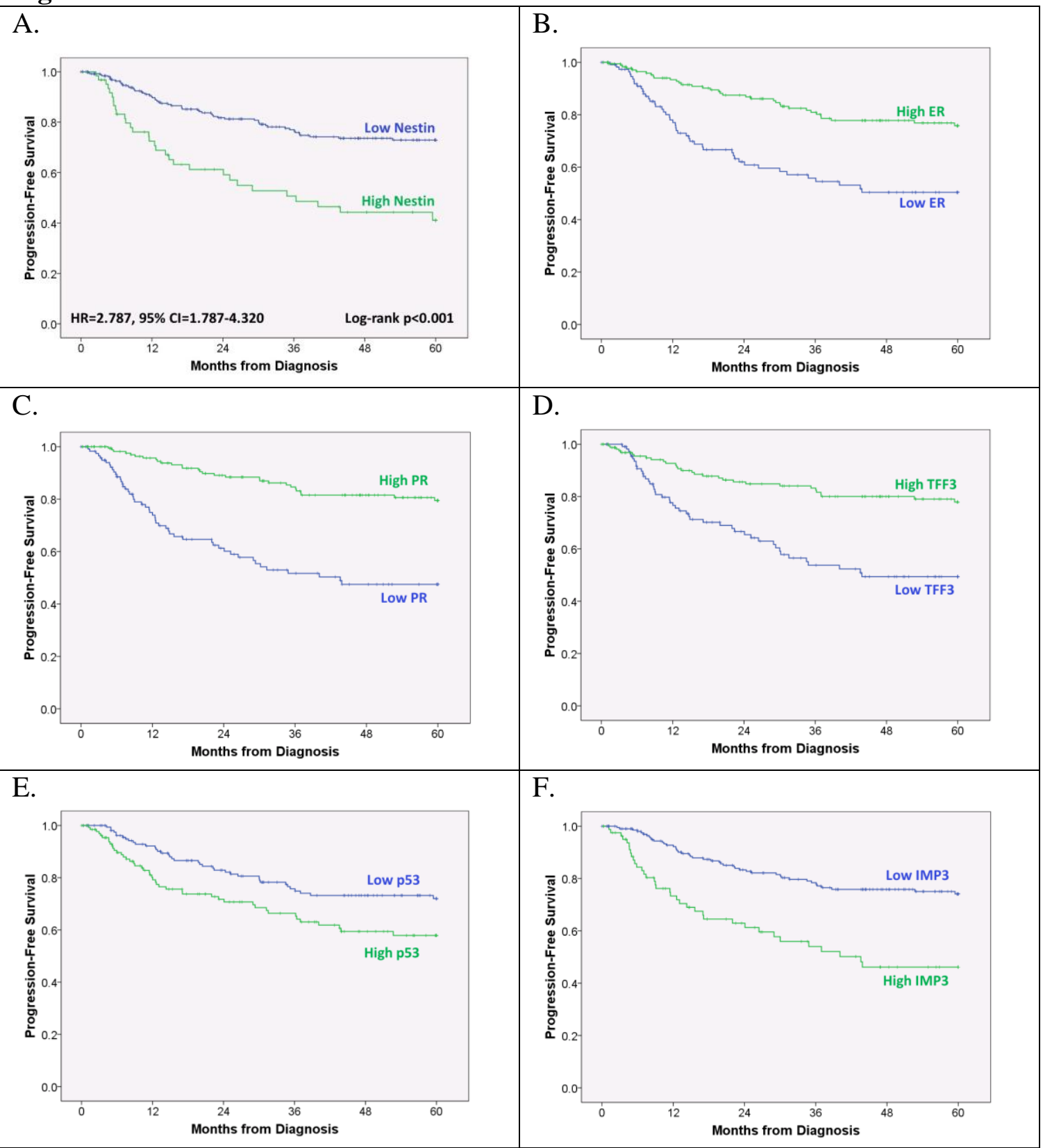


Hope et al. 35

Figure 3.
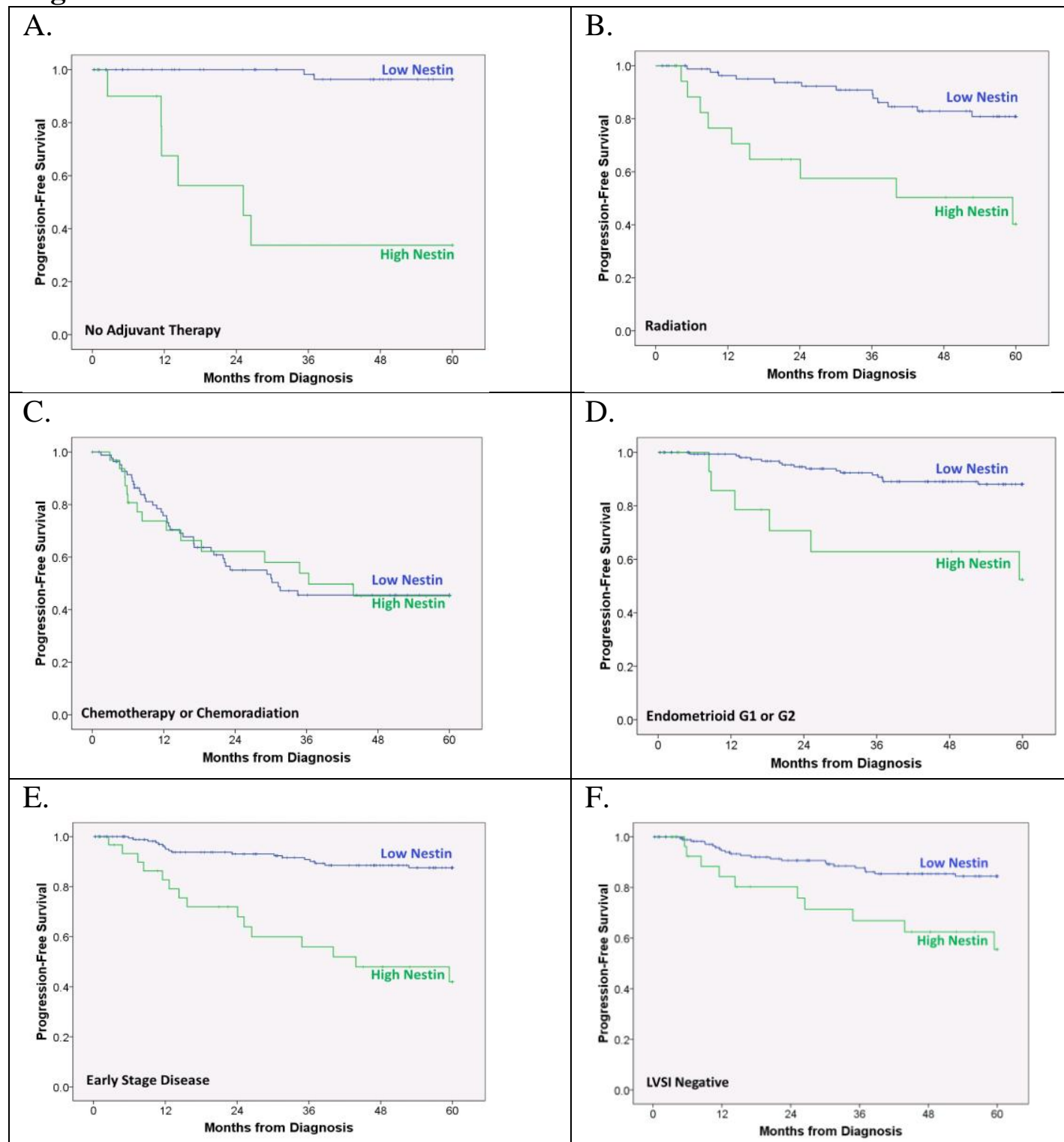

F.

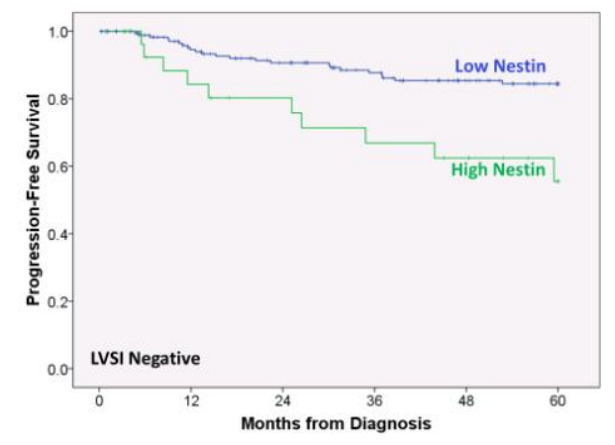

G.

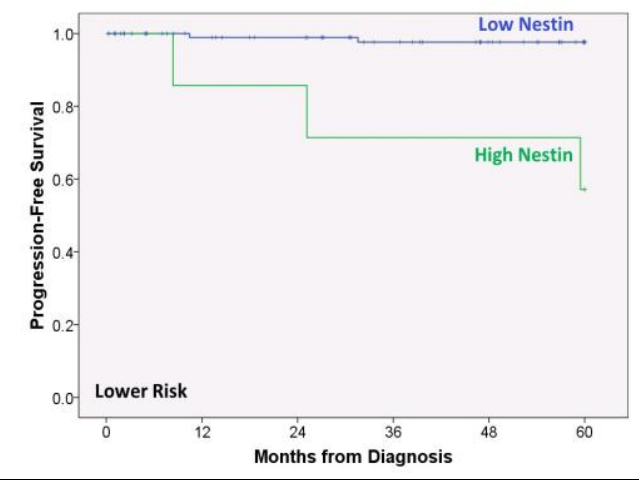

H.

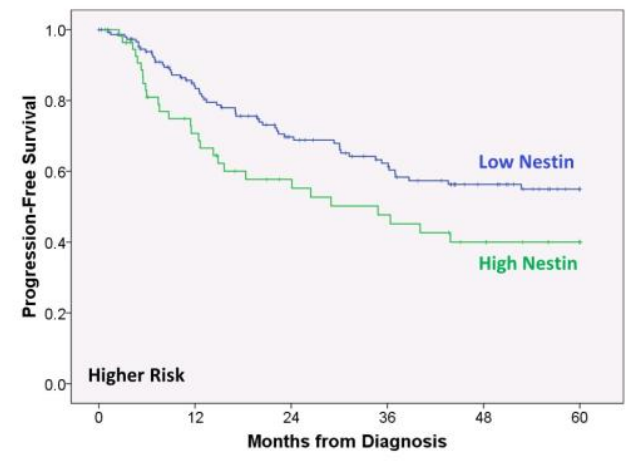


Figure 4.

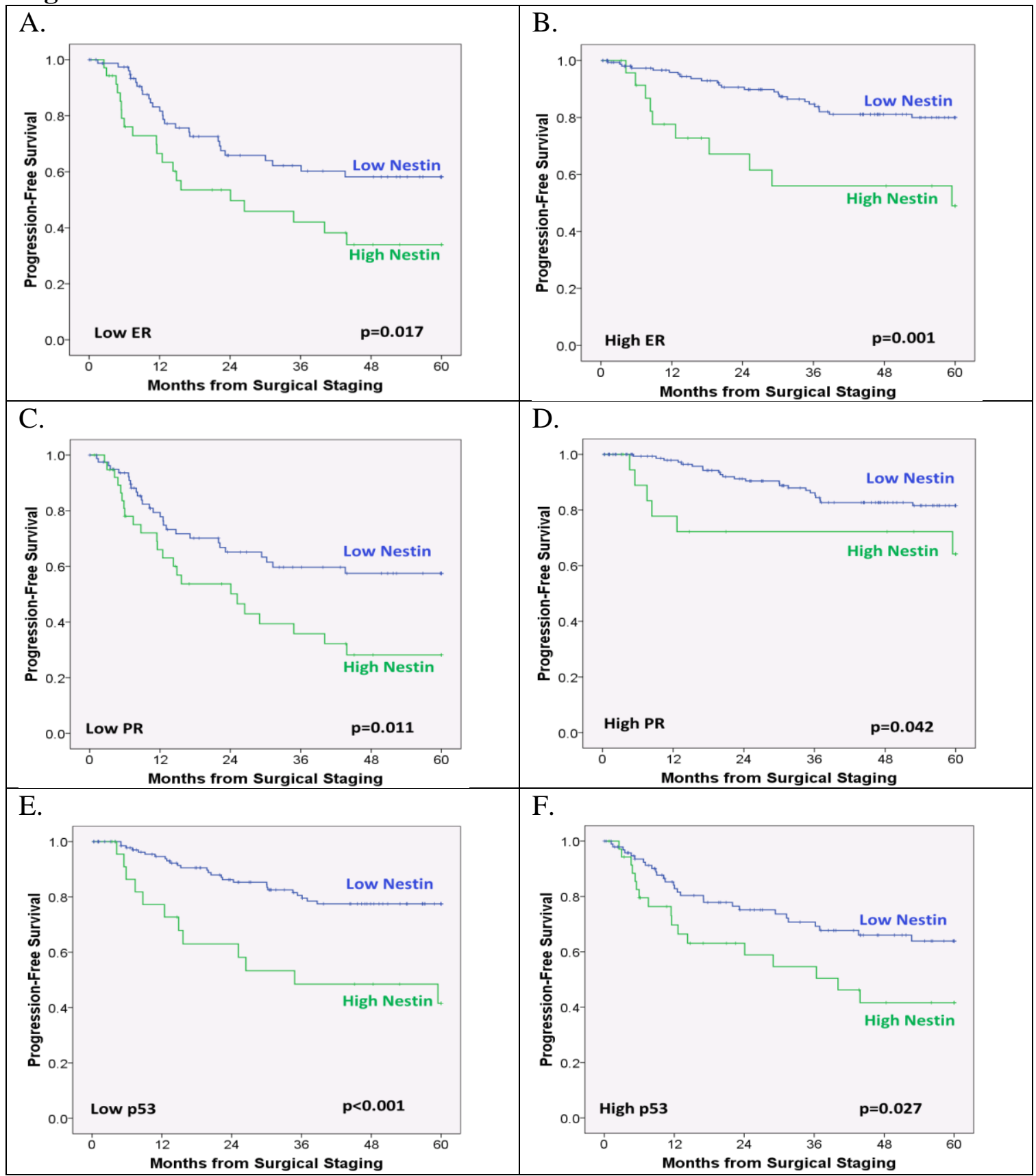

